\title{
Controlled radical polymerization of vinyl acetate in presence of mesoporous silica supported $\mathrm{TiCl}_{4}$ heterogeneous catalyst
}

\author{
M A SEMSARZADEH*, S AMIRI and M AZADEH \\ Tarbiat Modares University, Jalal Al-e-Ahmad Highway, Tehran, PO Box 14115-111, Iran
}

MS received 15 November 2011; revised 9 January 2012

\begin{abstract}
The heterogeneous $\mathrm{TiCl}_{4}$ catalysts supported on mesoporous mobile composition of matter (MCM-41) and mesoporous silicone particles synthesized from block copolymer of PPG-PEG-PPG (SPB) complexed with dimethyl formamide (DMF) ligand were used in a controlled free radical reaction with benzoyl peroxide (BPO) initiator in bulk polymerization of vinyl acetate (VAc). In this polymerization process, mesoporous particle of SPB increased the reactivity of $\mathrm{TiCl}_{4}$ catalyst with DMF ligand. The active site formed on the surface and the pores of the catalyst produced specific sequences of VAc on the chain with different thermal and microstructural properties and crystallinity.
\end{abstract}

Keywords. Mesoporous silica; heterogeneous catalyst; specific sequences; controlled free radical polymerization; crystallinity; vinyl acetate.

\section{Introduction}

In recent years, much interest has been generated by new techniques for controlled polymerization of monomers via free radical mechanisms. The controlled radical polymerization (CRP) allows for synthesis of polymers with unique architectures and for the relatively facile synthesis of block copolymers. CRP is a common technique for synthesis of (co)polymers with well-defined molecular parameters $\left(M_{\mathrm{w}} / M_{\mathrm{n}}\right)$, reactive end groups, composition and architecture. The most notable methodologies are atom transfer radical polymerization (ATRP), nitroxide-mediated polymerization (NMP or SFRP), and reversible addition-fragmentation chain transfer (RAFT) (Solomon et al 1985; Matyjaszewski and Xia 2001; Moad and Solomon 2006). Polyvinyl acetate(PVAc) is widely used in coatings industry. Moreover, it is important in industries as a precursor of polyvinyl alcohol. Therefore, molecular engineering of PVAc and development of suitable techniques are of utmost importance for production of novel polymeric materials. Unlike (meth) acrylates or styrene, vinyl acetate lacks conjugating substituent. Therefore, it is less reactive and difficult to polymerize. Also the sensitivity of vinyl acetate to oxygen has made its radical polymerization considerably more difficult to control (Kamigato et al 2001). In this process, the branching reactions compete with the free radical reaction. Solvent cage in the solution polymerization has been suggested to account for the reduced rate and diffusivity of the radicals, and therefore, higher branching of the polymer (Semsarzadeh et al 1997). The ATRP reactions of acrylates with vinyl acetate macroradicals are reported slowly in the solution and with

\footnotetext{
*Author for correspondence (semsarzadeh@modares.ac.ir)
}

definite induction times (Sandler 1992). The low yield of highly branched polymers reported was related to the random nature of the free radicals in vinyl acetate polymerization in the solution. Bounded radicals are known to control randomness of the reaction; and $\mathrm{NR}_{3}$ ligand used in the polymerization with the catalyst is reported to stabilize the complex in ethyl acetate radical polymerization of this monomer (Semsarzadeh et al 1997). Transition metals have recently been used to catalyze controlled radical polymerization of styrene and acrylates. A variety of transition metals such as copper, ruthenium, iron, cobalt, titanium and nickel have been successfully employed in ATRP (Kato et al 1995; Wang and Matyjaszewski 1995; Ando et al 1997; Matyjaszewski et al 1997; Kabachii et al 2003; Wang et al 2003). Alternatively, chlorine terminated vinyl acetate telomers has been used as a macroradical initiator to start atom transfer radical polymerization of vinyl acetate in block copolymerization reaction of vinyl acetate with methyl and methyl methacrylates or styrene (Kato et al 1995; Matyjaszewski et al 1997; Semsarzadeh and Abdollahi 2009; Semsarzadeh and Amiri 2012). To activate vinyl acetate monomer in the controlled radical polymerization, DMF ligand has been introduced with $\mathrm{Co}(a c a c)_{2}$ (Kaneyoshi and Matyjaszewski 2005). Other transition metal catalysts reported are $\mathrm{ZnCl}_{2}$ in DMF (Sandler 1992) and $\mathrm{SnCl}_{2}$ (Kaneyoshi and Matyjaszewski 2005). Later, acetoacetonate catalyst has been reported in the solution and bulk copolymerization of vinyl acetate with butyl acrylate (Debuigne et al 2008). The reactions reported have indicated an induction time of 7-10 h (Debuigne et al 2008). The effect of small quantity of DMF ligand on synthesized PVAc has been recently reported, which led to an increase in the rate and yield of this reaction (Kaneyoshi 
Table 1. Electronegativity of transition metals.

\begin{tabular}{lccccccccccc}
\hline & $\mathrm{Sc}$ & $\mathrm{Ti}$ & $\mathrm{V}$ & $\mathrm{Cr}$ & $\mathrm{Mn}$ & $\mathrm{Fe}$ & $\mathrm{Co}$ & $\mathrm{Ni}$ & $\mathrm{Cu}$ & $\mathrm{Zn}$ & $\mathrm{Sn}$ \\
\hline Electronegativity & 1.2 & 1.32 & 1.46 & 1.66 & 1.60 & 1.64 & 1.70 & 1.75 & 1.77 & 1.66 \\
Ionization potential & 6.54 & 6.83 & $6 \cdot 74$ & $6 \cdot 76$ & 7.43 & 7.90 & $7 \cdot 86$ & 7.63 & 7.72 & 9.93 \\
Electronics & $3 d^{1} 4 s^{2}$ & $3 d^{2} 4 s^{2}$ & $3 d^{3} 4 s^{2}$ & $3 d^{5} 4 s^{1}$ & $3 d^{5} 4 s^{2}$ & $3 d^{4} 4 s^{2}$ & $3 d^{4} 4 s^{2}$ & $3 d^{8} 4 s^{2}$ & $3 d^{2} 4 s^{1}$ & $4 d^{10} 5 s^{2}$ & $4 d^{10} 5 s^{2} 5 p^{2}$ \\
\hline
\end{tabular}

and Matyjaszewski 2005). In this paper, we report polymerization of vinyl acetate with heterogeneous catalysts of $\mathrm{TiCl}_{4}$. The mesoporous silica particles have been synthesized and used to make active heterogeneous $\mathrm{TiCl}_{4}$ catalyst to control the sequences of the growing chain in a controlled free radical polymerization of vinyl acetate. A small amount of DMF has been used as a ligand to activate the process. A more linear polymer was formed from the heterogeneous $\mathrm{TiCl}_{4}$ on the mesoporous SPB compared to MCM-41. The polymerization starts from the immobilized and fixed sites of the catalyst located on the surface or on the pores of this catalyst. The two centres in this polymerization produced two but different sequences of the polymer chain, each sequence indicating a different glass transition temperature with different $d$-spacings related to its own crystallinity.

\section{Theory of free radical reaction of vinyl acetate with $\mathrm{TiCl}_{4}$ silica heterogeneous catalyst in light}

According to our earlier reports, we considered the electronegativity of theoretically important transition metals in polymerization of vinyl acetate in light. In spite of reactivity of the catalysts in light in block copolymerization of styrene and acrylates, vinyl acetate has been reported as energetically unfavourable in block copolymerization with different monomers (Semsarzadeh and Abdollahi 2009; Semsarzadeh and Amiri 2012). The presence of active ligand as an electron donor has been reported to increase the reaction rate of vinyl acetate in light in the bulk (Kaneyoshi and Matyjaszewski 2005). Transition metals are reported to activate dormant species in controlled radical polymerization (Kaneyoshi and Matyjaszewski 2005; Asua 2007; Semsarzadeh and Aghili 2008). Certain transition metals increase the polymerization rate of vinyl acetate without any delay or induction time (Debuigne et al 2008). The electronic affinity and ionization of transition metals with their empty $d$ orbitals have been suggested to importantly contribute to this factor. The electronegativity of $\mathrm{Ti}(1.32)$ is far less than $\mathrm{TiCl}_{4}(1.7$ which has been suggested for polymerization in the bulk); therefore, under similar conditions, titanium as $\mathrm{TiCl}_{4}$ catalyst does not produce any polymer from vinyl acetate (Semsarzadeh et al 1997; Kamigato et al 2001; Semsarzadeh and Abdollahi 2009; Semsarzadeh and Amiri 2012). Apart from elctronegativity, electronic structures and crystalline geometry of catalysts may affect their reactivity or complex formations. In this work, we used a trace of DMF ligand to increase the reactivity of heterogeneous catalysts. The activated heterogeneous $\mathrm{TiCl}_{4}$ catalyst form starts free radical polymerization of vinyl acetate within a few hours. The reaction starts from two centres of immobilized active sites located on the surface and a pore of the catalyst, each centre activates the dormant free radicals from the controlled radical polymerization and triggers the growth of the chain in bulk polymerization of vinyl acetate with BPO (Kaneyoshi and Matyjaszewski 2005). The

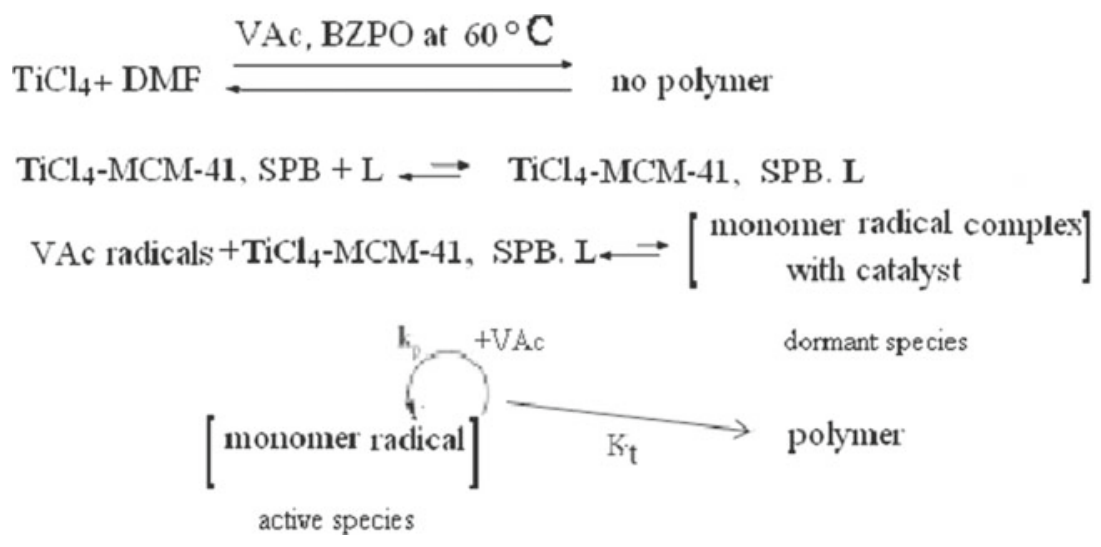

Scheme 1. Stereospecifically controlled free radical polymerization of VAc with mesoporous silica- $\mathrm{TiCl}_{4}$ catalysts. 
electronegativity and potentials of transition metals with their electronic structures used in bulk polymerization of vinyl acetate with PBO are shown in table 1 . The polymer chain grows in predetermined sequences initiated from these sites. The reaction then continued in a controlled linear fashion. The relatively low molecular weight distribution of about 2 (compared to 1.8 recently reported for block copolymers of vinyl acetate (Debuigne et al 2008) suggested a controlled mechanism similar to scheme 1 . In this mechanism, the active sites make a reversible complex with DMF ligand, which transfers the potentially random reactive species into the active monomer in a sequentially controlled stereospecific polymerization (reaction 1 of scheme 1 ). The active chain then grows on this sequence from the active catalyst of these two different centres to make a polymer chain with different microstructures consisting of two sequence units of polymer with crystallization temperature and higher melting point.

\section{Experimental}

\subsection{Materials}

Vinyl acetate (VAc) (Merck, >99\%) was dried over calcium hydride, distilled and stored under argon. Poly (propylene glycol)-poly (ethylene glycol)-poly (propylene glycol) (PPG-PEG-PPG, $M_{\text {avr }}=2700$ ) was purchased from SigmaAldrich. Tetraethyl orthosilicate (TEOS) was purchased from Acros. Moreover, $n$-hexadecyltrimethyl ammonium bromide (CTAB), aqueous ammonia (25 wt \%), $\mathrm{TiCl}_{4}, \mathrm{BPO}, \mathrm{DMF}$, citric acid and sodium citrate were all obtained from Merck and used without further purification.

\subsection{Preparation of catalyst supports}

MCM-41: The 2.4 g CTAB was first dissolved under mild stirring in $120 \mathrm{~g}$ deionized water at room temperature. Then $9.5 \mathrm{~g}$ ammonia solution was added slowly under stirring. Finally, $10 \mathrm{~g}$ of tetraethylorthosilicate was added dropwise for $15 \mathrm{~min}$. Next, the mixture was stirred for $1 \mathrm{~h}$, then the white precipitate was filtered, washed with deionized water for several times and dried in a vacuum oven at $90^{\circ} \mathrm{C}$ overnight. The final product was obtained by calcinations at $550{ }^{\circ} \mathrm{C}$ for $5 \mathrm{~h}$ in air (Saga and Terano 1994).

SPB: $\quad 0.5 \mathrm{~g}$ of PPG-PEG-PPG was dissolved in $48 \mathrm{~g}$ of deionized water after stirring at room temperature. Then $3.5 \mathrm{~mL}$ citric acid (1M) was added to the above solution. Subsequently, a mixture of $1 \mathrm{~mL} n$-octane and $12 \mathrm{~mL}$ ethanol was added to the emulsion under vigorous stirring. A milky emulsion formed immediately. After stirring for $1 \mathrm{~h}, 1.5 \mathrm{~mL}$ TEOS was added slowly. $\mathrm{pH}$ value was adjusted to 4.7 with $2 \mathrm{M} \mathrm{CH}_{3} \mathrm{COONa}$ solution. Next, when the white precipitate was produced, it was filtered and left in an oven at $80^{\circ} \mathrm{C}$ overnight. The final product was obtained by calcination at $550{ }^{\circ} \mathrm{C}$ for $5 \mathrm{~h}$ in air (Saga and Terano 1994).

\subsection{Polymerization of vinyl acetate}

The MCM-41 and SPB catalysts were treated in flowing ultra high-purity nitrogen at $400{ }^{\circ} \mathrm{C}$ for $9 \mathrm{~h}$ before use. The MCM41- and SPB-supported $\mathrm{TiCl}_{4}$ catalysts were synthesized according to the literature (Saga and Terano 1994). All polymerization were performed in dried glassware under argon and light at $60^{\circ} \mathrm{C}$ with a magnetic stirrer. Appropriate amount of the catalyst (SPB or MCM-41) was added to the system. A mixture of DMF, BPO and vinyl acetate was added (Catalyst: VAc: DMF: $\mathrm{BPO}=1 \cdot 20: 100: 19 \cdot 50: 0 \cdot 22)$. The volume ratio of monomer to DMF was 23. After 2, 3 and $4 \mathrm{~h}$, the obtained polymer was filtered, dissolved in methanol, precipitated in water and then dried at $60^{\circ} \mathrm{C}$ overnight (Kaneyoshi and Matyjaszewski 2005).

\subsection{Surface, structural and thermal characterizations}

The stereospecific polymerization of vinyl acetate strongly depends on the surface and solid structures of the mesoporous particles used in $\mathrm{TiCl}_{4}$ catalysts. The X-ray powder diffraction (XRD) spectra were recorded on a Philips X'pert diffractometer. X-ray fluorescence spectroscopy (XRF) was used to determine percent of titanium formed. The nitrogen adsorption-desorption isotherms were measured by BELSORP-mini adsorption apparatus. The specific surface areas of supports were measured by using BET (Brunauer, Emmett and Teller) method. ${ }^{1} \mathrm{H}$ NMR spectra were recorded with a Bruker DRX 500 spectrometer $(500 \mathrm{MHz})$ in deuterated chloroform. Infrared spectra were recorded with a Bruker IFS 48 FTIR spectrometer. Molecular weight and its distribution (MWD) were recorded with a Waters $150 \mathrm{C}$ PS-calibrated GPC in THF at $35^{\circ} \mathrm{C}$ and $1 \mathrm{~mL} /$ min equipped with a refractive index detector and 104, 103 and $500 \AA$ set of ultrastyrogel columns. Differential scanning calorimetery (DSC) analysis were recorded with a Pyris 1 instrument at a heating rate of $10^{\circ} \mathrm{C} / \mathrm{min}$ under nitrogen atmosphere in the range of -20 to $300^{\circ} \mathrm{C}$. Fourier transform infrared (FTIR) spectrum of inclusion complexes (solids) was recorded on an EQUINOX 55 Bruker FTIR Spectrophotometer.

\section{Results and discussion}

The heterogeneous polymerization of alkenes monomers with $\mathrm{TiCl}_{4}$ supported on $\mathrm{MgCl}_{2}$ is well documented in the literature (Saga and Terano 1994). Recently, mesoporous silica particles of MCM41 have been used in polymerization of ethylene (IUPAC Report 1985; Saga and Terano 1994; Dong et al 2006). The effects of high surface area and porosity of solid supports on increasing of reaction rates have been reported (Dong et al 2006). In this work results showed that vinyl acetate did not yield polymer with $\mathrm{DMF} / \mathrm{TiCl}_{4}$. Upon addition of $\mathrm{TiCl}_{4}$ to $\mathrm{DMF}$, a green solution was obtained, which represents reaction of the two components. After using $\mathrm{DMF} / \mathrm{TiCl}_{4}$ in polymerization, no polymer was formed after $8 \mathrm{~h}$. Then, $\mathrm{TiCl}_{4}$ supported on the mesoporous 

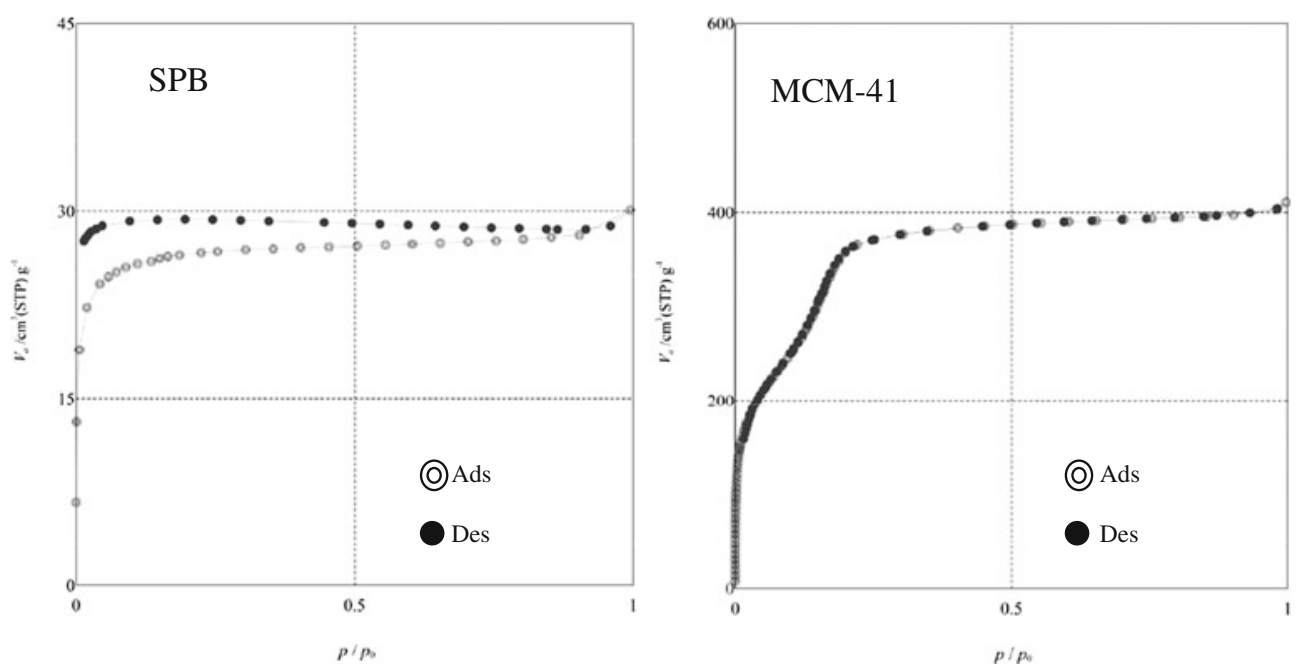

Figure 1. Nitrogen adsorption-desorption isotherm of MCM-41 and SPBS.

Table 2. Surface structures of MCM-41 and SPB particles ${ }^{\mathrm{a}}$.

\begin{tabular}{lccc}
\hline Support & $\mathrm{S}_{\mathrm{BET}}{ }^{\mathrm{b}}\left(\mathrm{m}^{2} / \mathrm{g}\right)$ & $\mathrm{V}_{\mathrm{p}}^{\mathrm{c}}(\mathrm{ml} / \mathrm{g})$ & $\mathrm{d}_{\mathrm{p}}{ }^{\mathrm{d}}(\mathrm{nm})$ \\
\hline MCM-41 & 1070 & 0.78 & 2.3 \\
SPB & 106 & 0.05 & 2.4 \\
\hline
\end{tabular}

${ }^{\text {a }} S_{\mathrm{BET}}$ is BET specific surface area; $V_{\mathrm{p}}$ is specific pore volume; $d_{\mathrm{p}}$, is pore diameter; ${ }^{\mathrm{b}}$ data from BET equation; ${ }^{\mathrm{c}}$ data from adsorption; ${ }^{\mathrm{d}}$ data from BJH calculation.

MCM-41 and SPB activated the monomer in polymerization in bulk. The nitrogen adsorption-desorption isotherms of MCM-41 and SPB supports were used to study the surface characteristics of the mesoporous particles (figure 1). The nitrogen adsorption-desorption isotherms of nitrogen of MCM-41 and SPB were of type IV according to the IUPAC nomenclature (Chokendoff and Nimantsverdrief 2007). It is worthy to note that adsorption and desorption isotherm curves of MCM-41 were superimposed completely, indicating that in this process, adsorption-desorption have the same energy. The same effect has been considered as a possible and known mechanism in the equilibrium formation (reaction 1) with heterogeneous polymerization (Seung et al 2001). The structural characteristics of MCM- 41 and SPB, including the specific surface areas, mean pore diameters and pore volumes used in this study, are shown in table 2 . The solid loadings of titanium used in synthesis of $\mathrm{TiCl}_{4}$ heterogeneous catalyst were measured by XRF as provided in table 3. Apart from different titanium loadings, the surface structures of the mesoporous silica supports with TEM and SEM have been reported earlier (Saga and Terano 1994). Since MCM-41 has a larger surface area than SPB, therefore, a higher number of $-\mathrm{OH}$ groups per mass unit of the supports was adsorbed for reaction with $\mathrm{TiCl}_{4}$ (table 3 ).
Table 3. Chemisorbed Ti on catalysts surface.

\begin{tabular}{lcc}
\hline Run & Catalyst & Ti content $(\%)$ \\
\hline 1. & ${\mathrm{MCM}-41-\mathrm{TiCl}_{4}}$ & $7 \cdot 23$ \\
2. & $\mathrm{SPB}-\mathrm{TiCl}_{4}$ & $3 \cdot 17$ \\
\hline
\end{tabular}

The conversion of vinyl acetate in bulk with MCM-41 and SPB-supported $\mathrm{TiCl}_{4}$ catalysts is shown in table 4 . The polymerization reactions continued without any delay and the conversion of polymer from $\ln (1 / 1-X)$ was linear with time (figure 2). Figure 3 shows linear reaction of vinyl acetate monomer in the controlled radical polymerization with $\mathrm{MCM}-41 / \mathrm{TiCl}_{4}$ and $\mathrm{SPB} / \mathrm{TiCl}_{4}$. The linear conversion rate, shown in figures 2 and 3, theoretically confirms a controlled radical polymerization. The relative degree of branching of the polymer from $\mathrm{TiCl}_{4} / \mathrm{MCM}-41$ and SPB catalysts was studied by ${ }^{1} \mathrm{HNMR}$ and the results indicated a higher degree of linearity for PVAc from $\mathrm{TiCl}_{4} / \mathrm{SPB}$ catalyst than $\mathrm{TiCl}_{4} / \mathrm{MCM}-41$. This effect was considered to be related to the specific surface structure of the heterogeneous $\mathrm{TiCl}_{4}$ catalysts (table 4). The specific surface area of MCM-41 was considerably higher than that of SPB (table 2). The surface studies of SPB and MCM-41 catalysts indicated that, in spite of the same pore size diameters, the surface area of SPB was less than MCM-41. Reactivity of the heterogeneous catalysts strongly depended on the surface area and pore size, where the active catalyst sites were originally formed and fixed. The higher percentage of Ti in MCM-41 was related to the higher existing specific surface area and pore volumes of the mesoporous solid particles. We used the specific [surface area/pore volume] ratio of the catalysts in this reaction. This ratio is higher for SPB particles (about 2120) compared to MCM-41 particles (1372). The pore diameters of MCM-41 and SPB are almost the same (about 2.3-2.7 nm). 
Table 4. Effect of mesoporous particles in $\mathrm{TiCl}_{4}$ on percent conversion, molecular weight and polydispersity of polymer in bulk polymerization of vinyl acetate at $60^{\circ} \mathrm{C}$.

\begin{tabular}{|c|c|c|c|c|c|c|}
\hline Run & Catalyst & Time (h) & Conversion (\%) & $M_{\mathrm{n}}\left(\mathrm{gmol}^{-1}\right)$ & $M_{\mathrm{w}} / M_{\mathrm{n}}$ & $\begin{array}{c}\text { Relative Branching } \\
\text { percent }(\%)^{\mathrm{a}}\end{array}$ \\
\hline \multirow[t]{3}{*}{1.} & $\mathrm{TiCl}_{4} / \mathrm{MCM}-41(0 \cdot 052)$ & 2 & 12 & 29500 & $3 \cdot 0$ & $1 \cdot 9$ \\
\hline & & 3 & 19 & 34000 & $2 \cdot 8$ & $1 \cdot 5$ \\
\hline & & 4 & 58 & 36600 & 2.78 & 1.4 \\
\hline \multirow[t]{3}{*}{2.} & $\mathrm{TiCl}_{4} / \mathrm{SPB}(0 \cdot 052)$ & 2 & 43 & 42900 & $2 \cdot 0$ & $1 \cdot 05$ \\
\hline & & 3 & 44 & 46700 & $1 \cdot 8$ & 0.9 \\
\hline & & 4 & 62 & 51200 & 1.78 & $0 \cdot 9$ \\
\hline
\end{tabular}

${ }^{\mathrm{a}}$ Calculated from ${ }^{1}$ HNMR (Datta et al 1996).
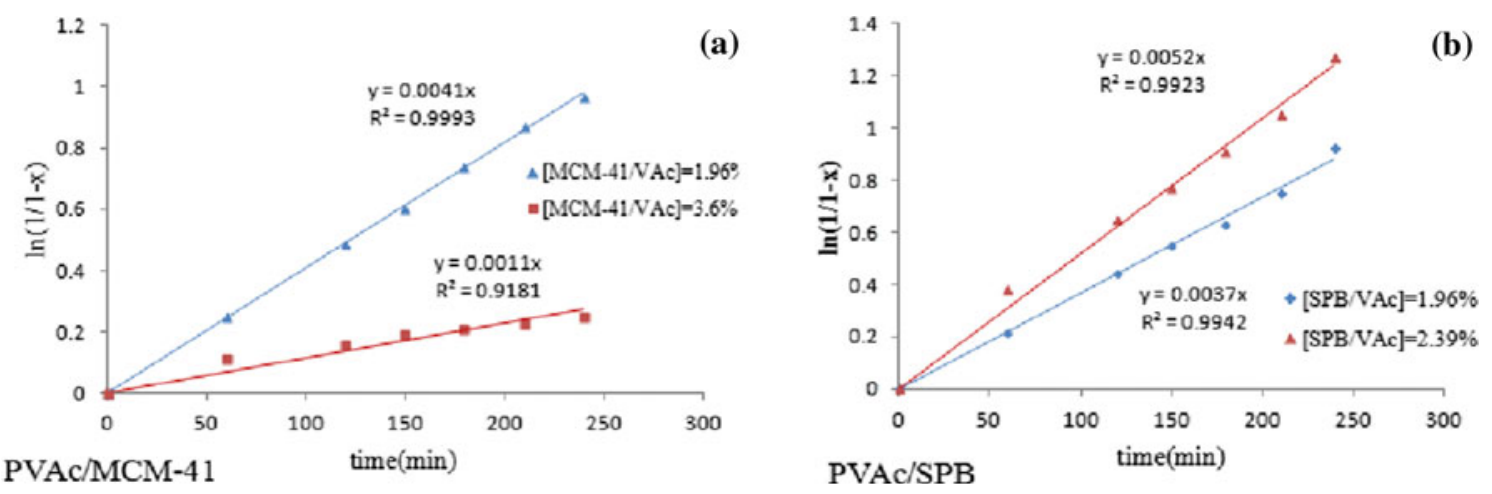

Figure 2. Semilogarithmic kinetic plots for bulk polymerization of vinyl acetate with (a) $\mathrm{SPB} / \mathrm{TiCl}_{4}$ and (b) $\mathrm{MCM} 41 / \mathrm{TiCl}_{4}$ at $60{ }^{\circ} \mathrm{C}$ with BPO.
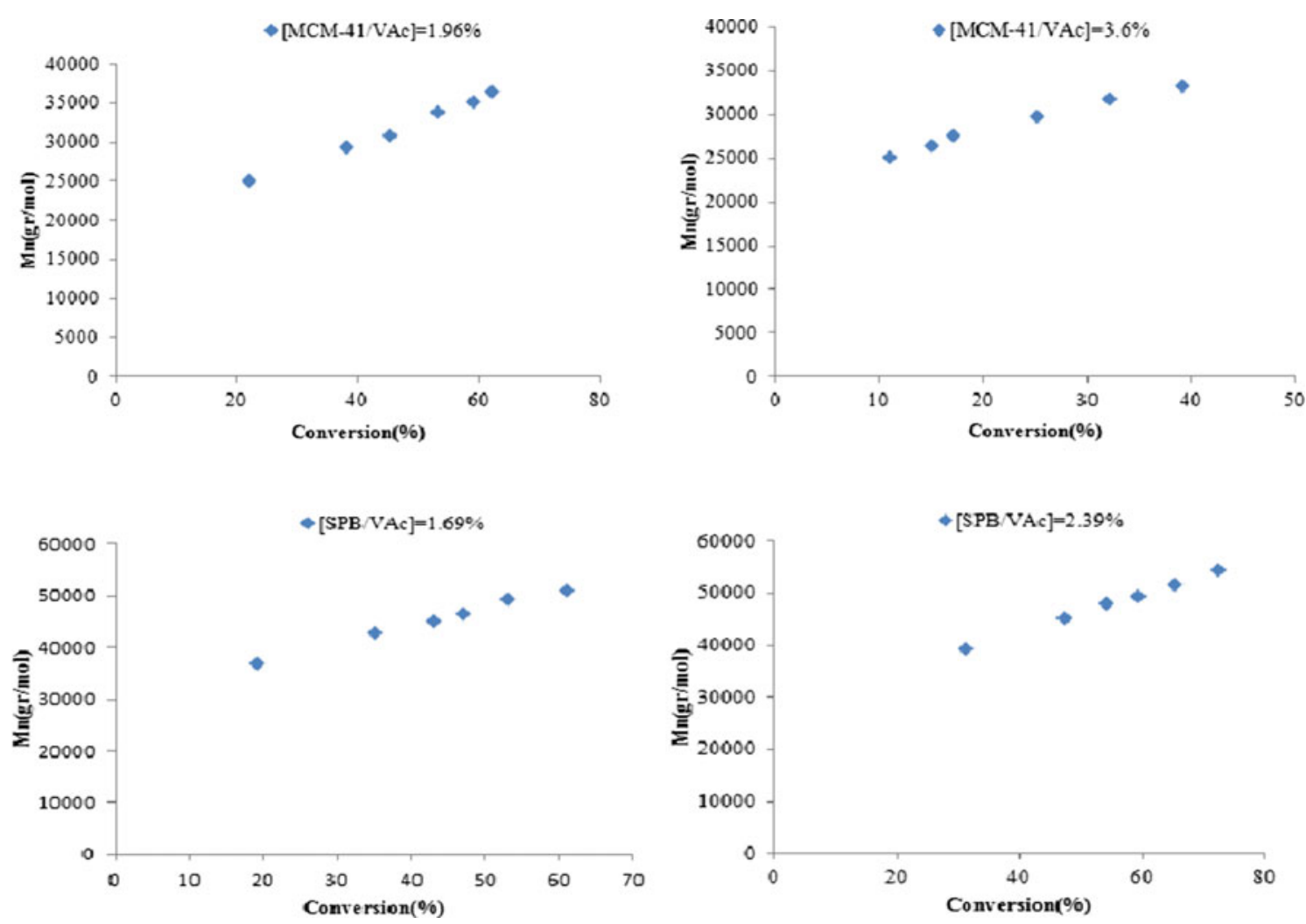

Figure 3. Dependence of polyvinyl acetate Mn on monomer conversion for polymerization initiated by BPO in DMF at $60{ }^{\circ} \mathrm{C}$ in bulk $\left([\mathrm{VAc}]_{0} /[\text { catalyst }]_{0} /[\mathrm{BPO}]_{0} /[\mathrm{DMF}]_{0}=83 \cdot 50 / 1 \cdot 00 / 0 \cdot 18 / 3 \cdot 85\right)$. 


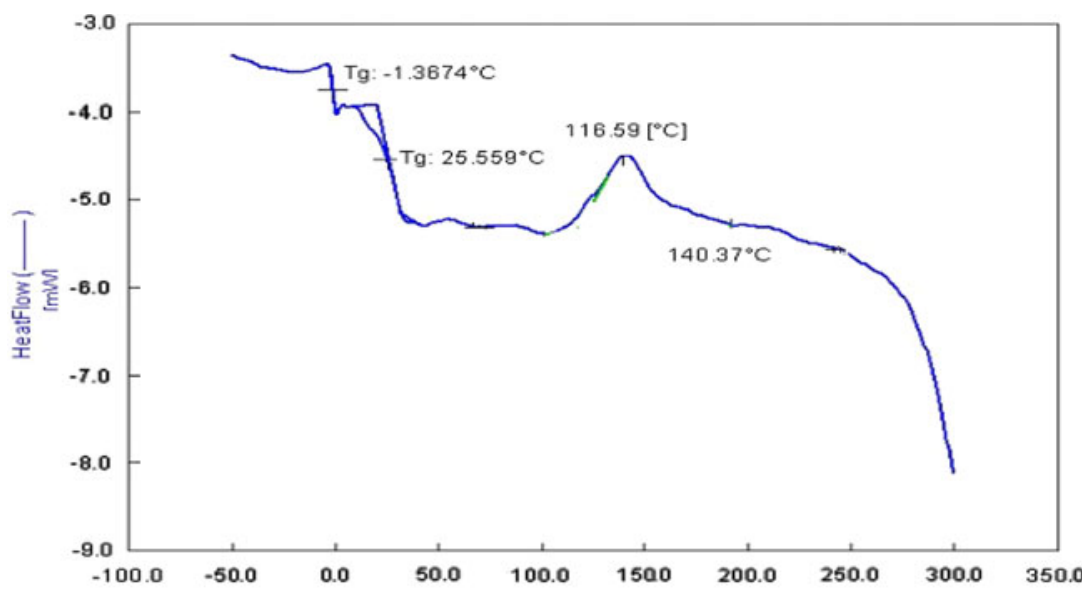

(a)

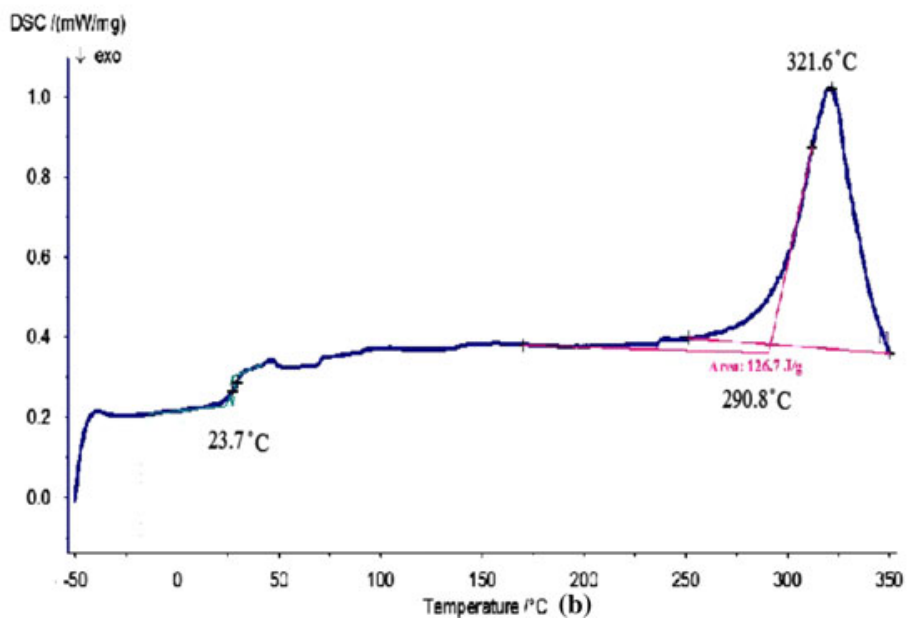

Figure 4. DSC for PVAc catalyzed by (a) $\mathrm{SPB} / \mathrm{TiCl}_{4}$ and (b) $\mathrm{MCM}-41 / \mathrm{TiCl}_{4}$ in bulk.

Thus, higher rate and reactivity of $\mathrm{TiCl}_{4} / \mathrm{SPB}$ catalyst with its particularly different crystalline and microstructural difference are related to the larger distribution of fixed active sites on the surface and pores of the particles, where two different sites were located to produce the specific sequences needed on the polymer chain. The equilibrium complex coordination of DMF ligand with each site of the $\mathrm{TiCl}_{4}$ particles activated the dormant radicals to continue in a predetermined sequence in a controlled radical polymerization. The distribution of $\mathrm{TiCl}_{4} / \mathrm{DMF}$ coordinated sites on the SPB surface and pores contributed to the sequentially different polymer chain.

In the absence of DMF ligand, PVAc conversion is much lower $\left(25 \%\right.$ after $6 \mathrm{~h}$ for $\left.\mathrm{MCM}-41 / \mathrm{TiCl}_{4}\right)$ than in the presence of DMF, because the catalyst/ligand complex helps continuous production of free radicals and keeps the system alive.

The transition temperature of polyvinyl acetate from the homogeneous catalysts of $\mathrm{Co}(a c a c)_{2}$ in the bulk has been reported (Kaneyoshi and Matyjaszewski 2005). The glass transition temperatures of polymer from the homogeneous catalysts are demonstrated in figure 4 . Thermal properties

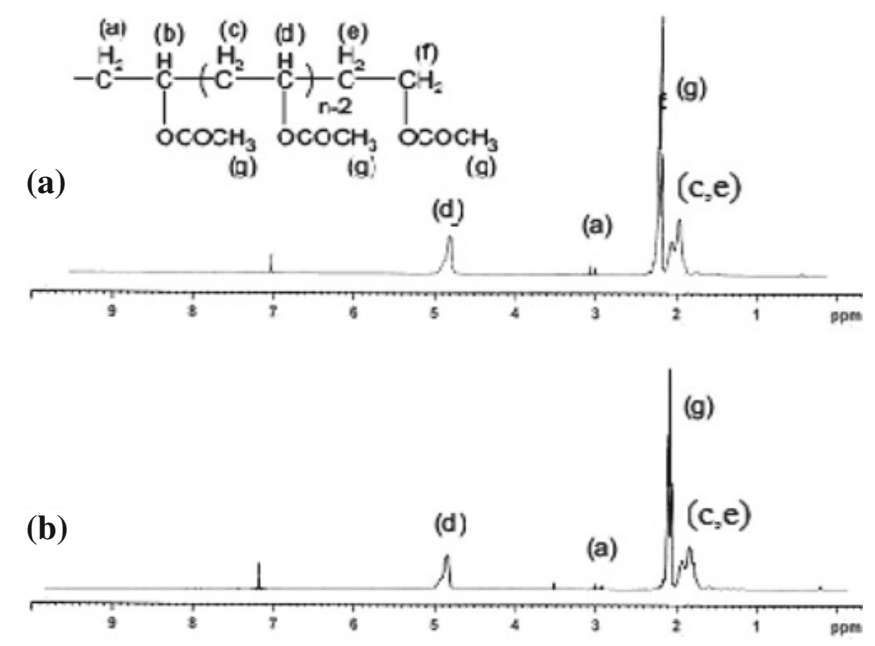

Figure 5. ${ }^{1} \mathrm{H}$ NMR spectrum of PVAc prepared using BZPO as initiator in presence of DMF ligand (a) $\mathrm{MCM}-41 / \mathrm{TiCl}_{4}$ and (b) $\mathrm{SPB} / \mathrm{TiCl}_{4}$ 
of polyvinyl acetate from these catalysts indicated different microstructures (Kaneyoshi and Matyjaszewski 2005). Lower glass transition temperatures at $-1{ }^{\circ} \mathrm{C}$ and $26^{\circ} \mathrm{C}$ of polyvinyl acetate from $\mathrm{TiCl}_{4}$ silicone particles indicated lower branching with two different sequences of polyvinyl acetate formed from two different sites of the catalysts in the polymerization reaction (figure 4). The crystallization temperature at $140^{\circ} \mathrm{C}$ was also related to the overall crystallinity of the polymer with its new microstructure before melting. DSC results indicated more crystalline structures for $\mathrm{PSB} / \mathrm{TiCl}_{4}$ than MCM-41/TiCl .
The ${ }^{1} \mathrm{HNMR}$ of PVAc prepared from the reaction of vinyl acetate with $\mathrm{MCM}-41 / \mathrm{TiCl}_{4}$ and $\mathrm{SPB} / \mathrm{TiCl}_{4}$ heterogeneous catalysts are shown in figure 5. The adsorption of methylene of PVAc from $\mathrm{TiCl}_{4} / \mathrm{MCM}-41$ and $\mathrm{TiCl}_{4} / \mathrm{SPB}$ catalysts indicated the adsorption at 1.4829-1.4779 ppm with a shift of about $0 \cdot 20-0.15 \mathrm{ppm}$. The methylene adsorption of the polymer made from $\mathrm{Co}(a c a c)_{2}$ catalyst is at $1.7233 \mathrm{ppm}$. The relative percentage of branching in the polymers from the heterogeneous catalysts was measured from the integral ratios at $[2 \cdot 86] /[1.93] \mathrm{ppm}$ of $\mathrm{CH}_{2}$ end group to $-\mathrm{OCH}_{3}$ of the chain. This ratio has been suggested

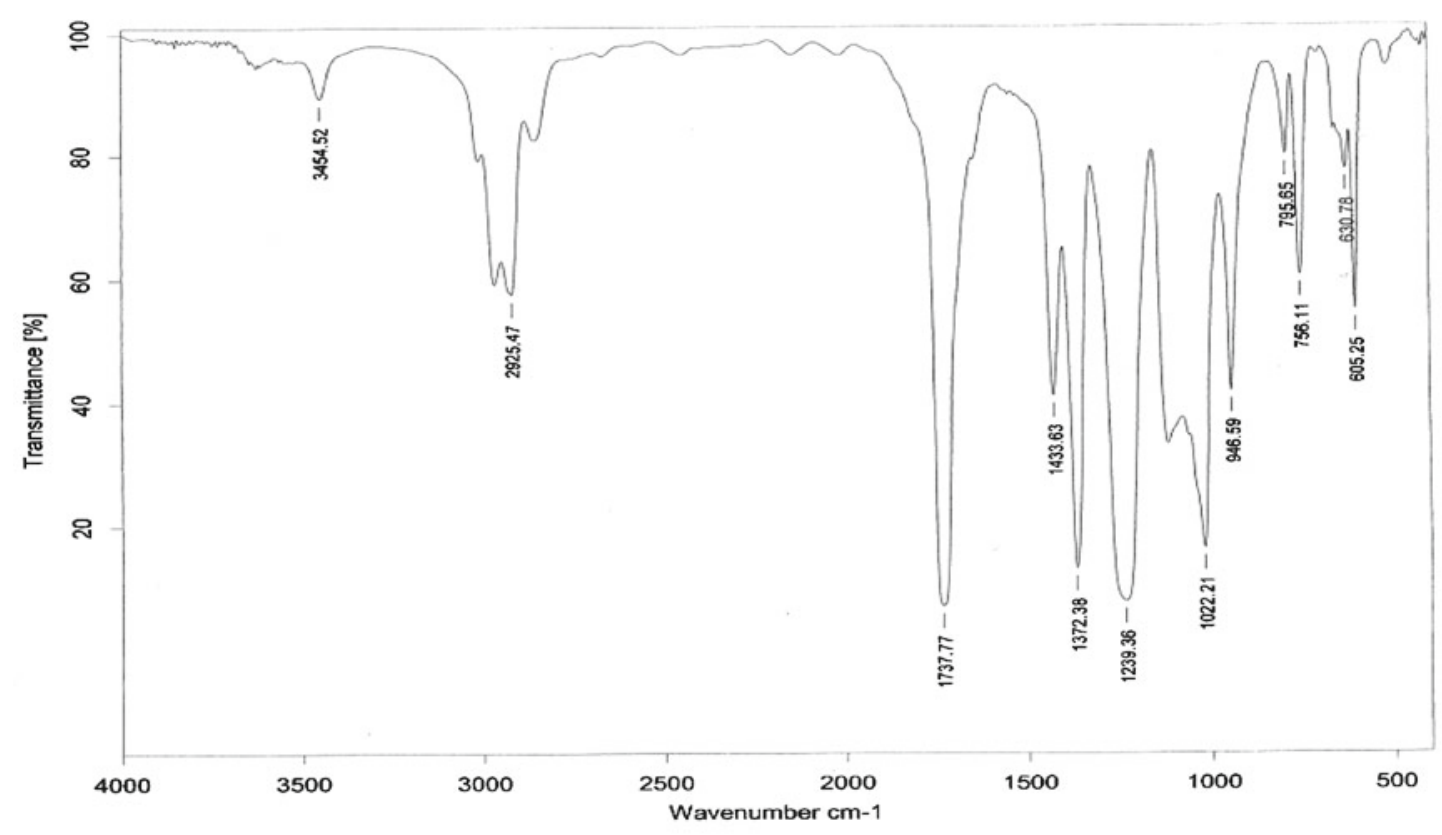

(a)

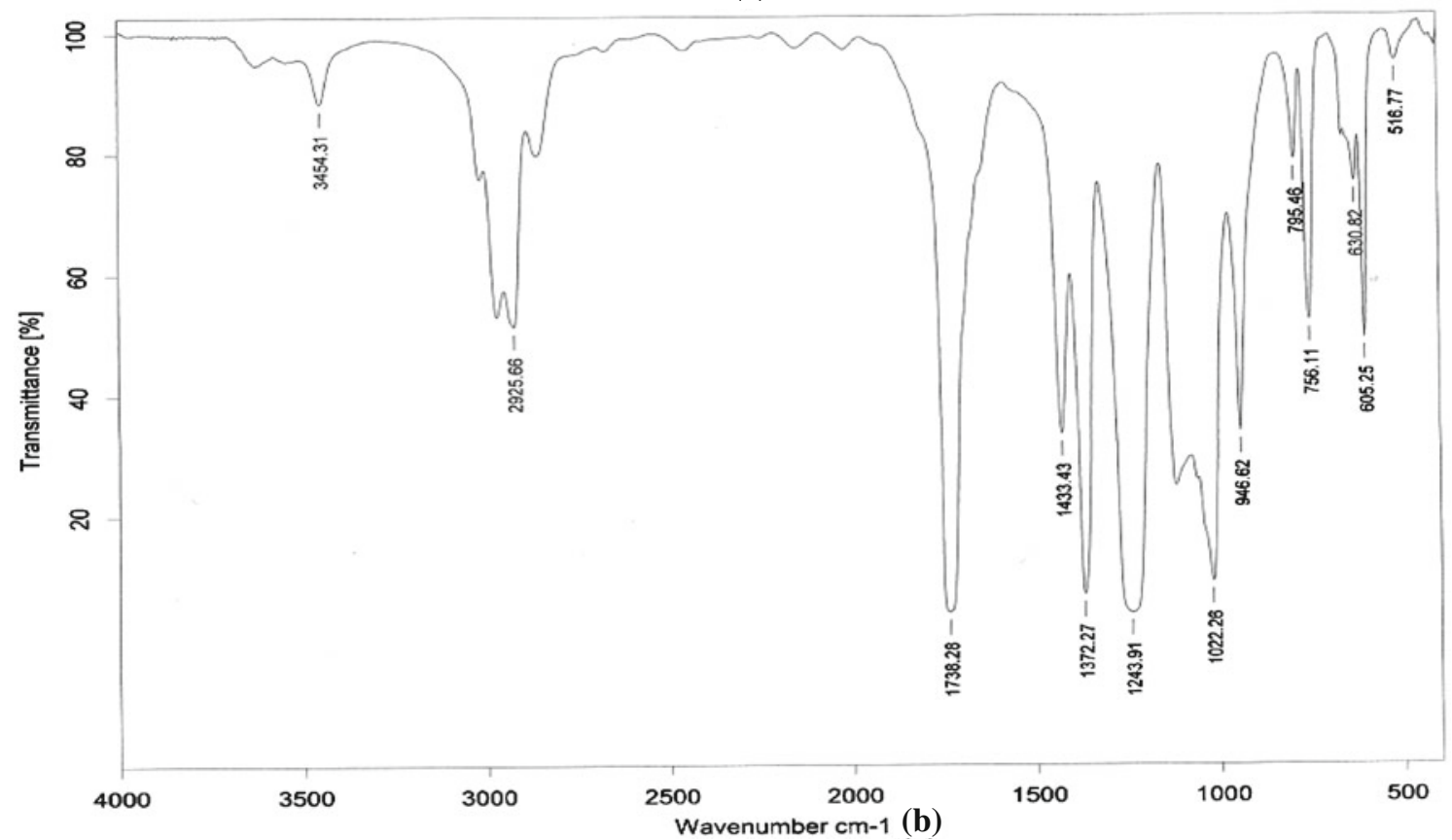

Figure 6. FTIR absorption spectra for PVAc catalyzed by (a) $\mathrm{SPB} / \mathrm{TiCl}_{4}$ and (b) $\mathrm{MCM}-41 / \mathrm{TiCl}_{4}$. 


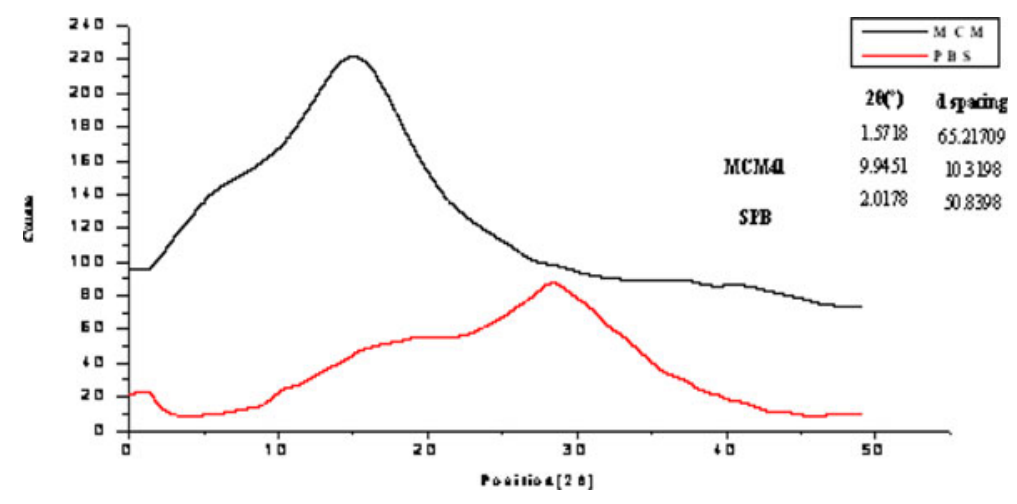

Figure 7. X-ray diffraction patterns of PVAc prepared by $\mathrm{MCM}-41 / \mathrm{TiCl}_{4}$ and $\mathrm{SPB} / \mathrm{TiCl}_{4}$ catalysts.

to indicate the relative degree of branching in polyvinyl acetate copolymers (Datta et al 1996). These results on the linear and crystalline microstructure of PVAc are compared well with the polydispersity index from GPC (table 4). The infrared structures of the polyvinyl acetate showed assigned peaks at $630.78 \mathrm{~cm}^{-1}$ (out-of-plane $\mathrm{C}-\mathrm{H}$ bend), $756 \cdot 11 \mathrm{~cm}^{-1}$ (in-plane $\mathrm{C}-\mathrm{H}$ bend), $1239.36 \mathrm{~cm}^{-1}(\mathrm{C}-\mathrm{CO}-\mathrm{C}$ bend), $1372.38 \mathrm{~cm}^{-1}$ (C-CO-C stretch), $1433.63 \mathrm{~cm}^{-1}\left(\delta_{\mathrm{s}}\right.$ $\left.\mathrm{CH}_{3}\right), 1737.77 \mathrm{~cm}^{-1}(\mathrm{C}=\mathrm{O}$ stretch $)$ and $2925.47 \mathrm{~cm}^{-1}\left(v_{\mathrm{s}}\right.$ $\mathrm{CH}_{2}$ ) (figure 6).

The microstructures of PVAc from $\mathrm{TiCl}_{4} / \mathrm{MCM}-41$ and SPB and the new crystalline peak catalysts which confirm the linear structure of PVAc are shown in figure 7. The presence of a broad shoulder with a peak at $2 \theta \cong 1.5^{\circ}$ and $9.94^{\circ}$ is assigned to polyvinyl acetate with $19.9 \%$ crystallinity from the polymerization reaction of vinyl acetate with $\mathrm{TiCl}_{4} / \mathrm{MCM}-41$ catalyst. The presence of this peak with $\mathrm{SPB} / \mathrm{TiCl}_{4}$ catalyst at $2 \theta \sim 2.01^{\circ}$ with the $d$-spacing of 50.8398 and increased crystallinity of $28 \%$ indicates a different microstructure related to the sequences formed during the polymerization of vinyl acetate under bulk.

\section{Conclusions}

MCM-41 and SPB were prepared with DMF ligand in the bulk polymerization of vinyl acetate with BPO initiator in atmospheric pressure. It was concluded that different microstructures were formed depending on the titanium and mesoporous silica particles used as support in these catalysts. The linear polymer in the presence of $\mathrm{TiCl}_{4} / \mathrm{SPB}$ catalyst has been specifically formed from the immobilized and fixed sites located in the surface and pores of the catalysts involved in the contact of monomer with the surface or diffusions of monomer into the pores of the catalysts. During polymerization, two different sites react and produce two different sequences in the polyvinyl acetate chain. In this case, each sequence is controlled to produce a microstructure with a higher degree of crystallinity, and crystalline and melting temperatures.

\section{References}

Ando T, Kamigaito M and Sawamoto M 1997 Macromolecules 30 4507

Asua J M 2007 Polymer reaction engineering (UK: Blackwell)

Chokendoff I and Nimantsverdrief J W 2007 Concepts of modern catalysis and kinetics 2nd, Revised and Enlarged Edition (Wiley) pp. 56-63, 207-216

Datta S K, Bhowmick A K, Chaki T K, Majali A B and Dehpande R S 1996 Polymer 3745

Debuigne A, Michaux C, Jerôme C, Jerôme R, Poli R and Detrembleur C 2008 Chem. Eur. J. 147623

Dong X, Wang L, Zhou J, Yu H and Sun T 2006 Catal. Commun. 71

IUPAC Report 1985 Pure. Appl. Chem. 57603

Kabachii Y A, Kochev S Y, Bronstein L M, Blagodatskikh I B and Valetsky P M 2003 Polym. Bull. 50271

Kamigato M, Ando T and Sawamoto M 2001 Chem. Rev. 1013689

Kaneyoshi H and Matyjaszewski K 2005 Macromolecules 388163

Kato M, Kamigaito M, Sawamoto M and Higashimura T 1995 Macromolecules 281721

Matyjaszewski K and Xia J 2001 Chem. Rev. 1012921

Matyjaszewski K, Wei M, Xia J and McDermott N E 1997 Macromolecules 308161

Moad G and Solomon D H 2006 The chemistry of radical polymerization (Oxford, UK: Elsevier) 2nd ed.

Saga K and Terano M 1994 Catalyst design for tailor made polyolefines (Elsevier)

Sandler S R 1992 Polymer synthesis (New York: Academic Press) 2nd ed.

Semsarzadeh M A and Abdollahi M 2009 J. Appl. Polym. Sci. 114 2509

Semsarzadeh M A and Aghili A 2008 J. Macromol. Sci. 45680

Semsarzadeh M A and Amiri S 2012 Controlled free radical polymerization of vinyl acetate with cobalt acetoacetonate. J. Chem. Sci. 124(2) 521-527

Semsarzadeh M A, Karimi A and Eshtad M 1997 Iranian Polym. J. 6261

Seung H Y, Tae H Y and Jae Y J 2001 Macromol. Rapid Commun. 221319

Solomon D H, Rizzardo E and Cacioli P 1985 European Patent Application 63

Wang J S and Matyjaszewski K 1995 J. Am. Chem. Soc. 1175614

Wang B, Zhuang Y, Luo X, Xu S and Zhou X 2003 Macromolecules 369684 